\title{
On Domains of Dependence and Partial Lacunas for Symmetric Hyperbolic Systems
}

\author{
G. F. D. DUFF \& A. TSUTSUMI
}

Communicated by P. GaRABEDIAN

\section{Contents}

1. Introduction. . . . . . . . . . . . . . . . . . . . . . . . 219

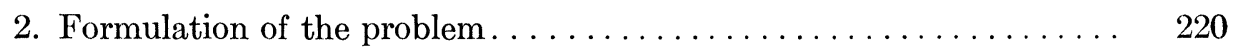

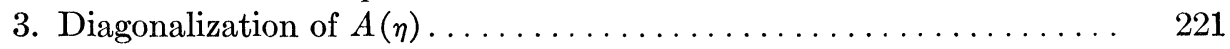

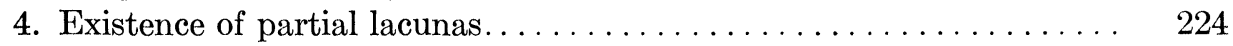

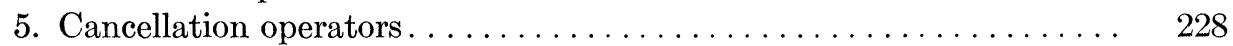

6. Supports and singular supports....................... 229

7. Example: Maxwell's equations...................... 232

1. Introduction. A distinctive feature of equations or systems of the hyperbolic type is that the domains of dependence of their solutions are bounded. For linear systems with constant coefficients this property is embodied in the behaviour of the fundamental matrix solution, which at any time subsequent to the initial instant has bounded support. The singular support of such an elementary solution is the wave surface associated with the system, of which the outermost sheet also bounds the support.

For hyperbolic equations of higher order with constant coefficients, the phenomenon of lacunas has been widely studied: [2], [3], [7], [10], [11], [12], [15], [17], [18], [20], [22]. A lacuna is a region bounded by (piecewise analytic) sheets of the wave surface that does not belong to the support of the fundamental solution, or (what is the same thing) to the domain of dependence. In this paper we study the lacunas associated with a first order symmetric hyperbolic system with constant coefficients, and show that in general lacunas are present for certain linear combinations of derivatives of the solution components. These combinations, which we call differential lacunary components, each have domains of dependence related to one of the irreducible factors of the characteristic or 
determinental polynomial of the system. Usually the investigations on lacunas are based on the expression or formula for the solution, but to establish the existence and properties of the differential lacunary components (abbreviated d.l.c.) we proceed here as follows. We introduce the symmetric hyperbolic system with constant coefficients, and study the algebra related to its wave surface, particularly the irreducible characteristic factors and their multiplicities, and the eigenvectors of the general coefficient matrix. The d.l.c. are then constructed and shown to satisfy an appropriate polynomial equation of hyperbolic type related to the characteristic factor polynomials. From these equations the domain of dependence and lacunary properties are deduced. A basis theorem is established, involving the solution of a related system to obtain the solution components given the d.l.c. An irreducibility or minimality theorem for the d.l.c. is also proved. Examples are given in connection with Maxwell's, the acoustic and Dirac equations (see [4], p. 176).

The existence of d.l.c. and of the consequent partial lacunas was shown by one of the authors in an earlier report [6], for the particular case of simple characteristic factors. The present generalization to higher multiplicities is, however, relevant even to such immediate examples as Maxwell's equations.

2. Symmetric hyperbolic systems. The existence and uniqueness theorems for the Cauchy problem for hyperbolic differential equations is well known [19], although the corresponding theorems for first order symmetric hyperbolic systems were published as recently as 1954 by Friedrichs [8]. The lacunas for single equations were studied in a remarkable paper by Petrowsky [18].

Since an arbitrary solution of a symmetric hyperbolic system can be obtained by convolution with the elementary solution, we shall in the following consider primarily the elementary solution (or fundamental matrix solution). As the supports and singular support of the elementary solution are directly related to the wave surfaces (see [7], p. 19, [4], p. 587) $W_{l}(x, t)$ of the factor polynomials, the discussion of these supports then takes the simplest possible form.

Consider an $N$-dimensional Euclidean space $E_{N}$ of variables $x_{\nu}, \nu=1, \cdots, N$, and let $N$ square matrices $A_{\nu}$ of order $m$, with constant components $A_{p q}^{v}$, be given. We denote time by $t$, and prescribe the system of differential equations with constant coefficients

$$
\frac{\partial u}{\partial t}=\sum_{\nu=1}^{N} A_{\nu} \frac{\partial u}{\partial x_{\nu}}
$$

where $u$ is a $m$-component vector $u(t, x)$. We assume that (2.1) is symmetric hyperbolic:

$$
A_{i}, \quad i=1,2, \cdots, N, \text { are Hermitian matrices. }
$$

Let $\delta(\lambda)$ be

$$
\delta(\lambda)=A(\eta)-\lambda E
$$


where $A(\eta)=\sum_{\nu=1}^{N} A_{\nu} \eta_{\nu}, \eta=\left(\eta, \cdots, \eta_{N}\right)$ and $E$ is the unit matrix of order $m$. We note that $A(\eta)$ is homogeneous of degree one with respect to $\eta$. Define

$$
\Delta(\lambda)=\operatorname{det} \delta(\lambda) .
$$

It follows from (2.2) that all roots of $\Delta(\lambda)=0$, namely the eigenvalues of $A(\eta)$, are real for real vectors $\eta . \Delta(\lambda)$ is a product of irreducible factors of lower degree

$$
Q_{1}(\lambda, \eta) Q_{2}(\lambda, \eta) \cdots Q_{L}(\lambda, \eta)
$$

which are polynomials in $\lambda$ and $\eta$, and we allow repeated factors among them. That implies that we can write $\Delta(\lambda)$ in the form

$$
\left\{P_{1}(\lambda, \eta)\right\}^{\gamma_{1}}\left\{P_{2}(\lambda, \eta)\right\}^{\gamma_{3}} \cdots\left\{P_{l}(\lambda, \eta)\right\}^{\gamma_{l}} \cdots\left\{P_{k}(\lambda, \eta)\right\}^{\gamma_{k}},
$$

where the roots of $P_{l}(\lambda, \eta)=0, \lambda_{1}^{(l)}, \cdots, \lambda_{m l}^{(l)}$, are all simple.

3. Diagonalization of $A(\eta)$. First we shall find the eigenvectors of $A(\eta)$ for an eigenvalue $\lambda$.

Lemma 3.1. For a $\lambda_{\mu}^{(l)}, 1 \leqq \mu \leqq m_{l}$, there exist $\gamma_{l}$ linearly independent eigenvectors $T_{l \mu}^{(\nu)}, \nu=1,2, \cdots, \gamma_{l}$, whose components are homogeneous polynomials in $\lambda_{\mu}^{(l)}, \eta$ of degree $m-\gamma_{l}$ not identically zero on $|\eta|=1$.

Here "linearly independent" means linearly independent as polynomials in $\lambda_{\mu}^{(l)}$ and $\eta$.

Proof. As $\lambda_{\mu}^{(l)}$ is a root of $\Delta(\lambda)=0$ with the multiplicity $\gamma_{l}$, the rank of $\delta\left(\lambda_{\mu}^{(l)}\right)$ is $m-\gamma_{l}$. Hence for a fixed $\eta$ on $|\eta|=1$, there exists a minor of order $m-\gamma_{l}$ of $\delta\left(\lambda_{\mu}^{(l)}\right)$ whose determinant is not zero. Without loss of generality we can express it in the form

$$
\mathfrak{A}=\left(b_{i i}^{(l, \mu)}: \begin{array}{l}
i \downarrow 1,2, \cdots, m-\gamma_{l} \\
j \rightarrow 1
\end{array}\right), \quad|\mathfrak{M}|=\operatorname{det} \mathfrak{A} \neq 0 .
$$

Next we set

$$
\begin{aligned}
\mathscr{L} & =\left(b_{i i}^{(l, \mu)}: \begin{array}{c}
i \downarrow 1,2, \cdots, m-\gamma_{\iota} \\
j \rightarrow m-\gamma_{l}+1, \cdots, m
\end{array}\right), \\
S_{l, \mu}^{(1)} & =\left(S_{i} \cdot i \downarrow 1,2, \cdots, m-\gamma_{l}\right) \text { and } \\
S_{l, \mu}^{(2)} & =\left(S_{i} \cdot i \downarrow m-\gamma_{l}+1, \cdots, m\right) .
\end{aligned}
$$

We can solve with Cramer's formula the linear algebraic system

$$
\mathfrak{A} S_{i, \mu}^{(1)}=-\mathfrak{L} S_{l, \mu}^{(2)}
$$

for $S_{l, \mu}^{(2)(\nu)}=C_{l, \mu}^{(\nu)}=\left(S_{i}: S_{i}=1, i=\nu, S_{i}=0, i \neq \nu, i \downarrow m-\gamma_{l}+1, \cdots, m\right)$. Let the solution be denoted by

$$
S_{l, \mu}^{(1)(\nu)} \quad \text { and let } \quad S_{l, \mu}^{(\nu)}=\left(\begin{array}{l}
S_{l, \mu}^{(1)(\nu)} \\
S_{l, \mu}^{(2)(\nu)}
\end{array}\right) .
$$


We then set

$$
T_{l, \mu}^{(\nu)}=|\mathfrak{A}| S_{l, \mu}^{(\nu)}=\left(T_{i} \cdot i \downarrow 1,2, \cdots, m\right) .
$$

Thus

$$
T_{s}=\operatorname{det}\left(b_{i j}^{\prime}: b_{i j}^{\prime}=b_{i j}, j \neq s, b_{i j}^{\prime}=b_{i \nu}, j=s, \begin{array}{l}
i \downarrow 1,2, \cdots, m-\gamma_{\imath} \\
j \rightarrow 1,2, \cdots, m-\gamma_{\iota}
\end{array}\right)
$$

for $s=1,2, \cdots, m-\gamma_{l}$, and $T_{s}=0, s \neq \nu, T_{s}=-|\mathfrak{A}|, s=\nu$ for $s=m-$ $\gamma_{l}+1, \cdots, m$. For $\nu=m-\gamma_{l}+1, \cdots, m$ in (3.1) we get $\gamma_{l}$ linearly independent solutions of

$$
\delta\left(\lambda_{\mu}^{(l)}\right) T_{l, \mu}^{(\nu)}=0 .
$$

This proves Lemma 1, because the components of (3.1) are the homogeneous polynomials in $\lambda_{\mu}^{(l)}$ and $\eta$ of degree $m-l$ while $T_{l, \mu}^{(\nu)}$ is not identically zero on $|\eta|=1$. Now we rewrite (3.1) as follows:

$$
\begin{aligned}
T_{\mu, l}^{(\nu)} & =T_{l}^{(\nu)}\left(\lambda_{\mu}^{(l)}, \eta\right) \\
& =\left(T_{l i}^{(\nu)}\left(\lambda_{\mu}^{(l)}, \eta\right) \cdot i \downarrow 1,2, \cdots, m\right), \quad \nu=1,2, \cdots, \gamma_{l} .
\end{aligned}
$$

Lemma 3.2. For any other eigenvalue $\lambda_{\mu^{\prime}}^{(l)}, \mu^{\prime} \neq \mu, 1 \leqq \mu^{\prime} \leqq m_{l}$, the l linearly independent eigenvectors of $A(\eta)$ are constructed merely by replacing $\lambda_{\mu}^{(l)}$ in (3.3) by $\lambda_{\mu^{\prime}}^{(l)}$.

Proof. First we shall review the mechanism by which the left hand side of (3.2) is a zero vector. By (3.1) we can calculate the $i$ th row of (3.2) for $i=$ $1,2, \cdots, m-l$ as follows:

$$
\sum_{s=1}^{m} b_{i s} T_{s}=\sum_{s \neq \nu} b_{i s} T_{s}+b_{i \nu} T_{\nu}=\sum_{s \neq \nu} b_{i s}(-1)^{m-\gamma_{l}-s} \cdot \operatorname{det}\left(b_{i j}^{\prime \prime}\right)-b_{i \nu}|\mathfrak{A}|,
$$

where $b_{i j}^{\prime \prime}$ are equal to $b_{i j}(1 \leqq j \leqq s-1), b_{i, j+1}\left(s+1 \leqq j \leqq m-\gamma_{l}-1\right)$. and $b_{i \nu}\left(j=m-\gamma_{l}\right)$ for $i \downarrow 1,2, \cdots, m-\gamma_{l}$. Hence we have

$$
\sum_{s=1}^{m} b_{i s} T_{s}=-\operatorname{det}\left(b_{i j}^{\prime \prime \prime}\right) \text {, }
$$

where $b_{i j}^{\prime \prime \prime}=b_{i j}\left(1 \leqq i \leqq m-\gamma_{l}, 1 \leqq j \leqq m-\gamma_{l}\right), b_{i, m-\gamma_{l}+1}^{\prime \prime \prime}=b_{i v}(1 \leqq$ $\left.i \leqq m-\gamma_{l}\right)$ and $b_{m-\gamma l+1, j}^{\prime \prime \prime}=b_{i j}\left(1 \leqq j \leqq m-\gamma_{l}+1\right)$. Therefore this determinant is 0 . Note the two equal rows; namely the $i$-th and $m-\gamma_{l}+1$-st rows. This is independent of $\lambda_{\mu}^{(l)}$ being a characteristic root, which holds even after replacing $\lambda_{\mu}^{(l)}$ by $\lambda_{\mu^{\prime}}^{(l)}$ in (3.3). For $i=m-\gamma_{l}+1, \cdots, m$,

$\sum_{s=1}^{m} b_{i s} T_{s}=-\operatorname{det}\left(b_{i j}^{\prime \prime \prime}\right)$, where $b_{i j}^{\prime \prime \prime}=b_{i j}\left(1 \leqq i \leqq m-\gamma_{l}, 1 \leqq j \leqq m-\gamma_{l}\right)$, $b_{i, m-\gamma l+1}^{\prime \prime \prime}=b_{i v}\left(1 \leqq i \leqq m-\gamma_{l}\right)$ and $b_{m-\gamma_{l+1, j}}^{\prime \prime \prime}=b_{i j}\left(1 \leqq j \leqq m-\gamma_{l}+1\right)$ is the minor of $\delta\left(\lambda_{\mu}^{(l)}\right)$ of order $m-l+1(\leqq m)$, and hence is zero since $\lambda_{\mu}^{(l)}$ is 
a root of $\Delta(\lambda)=0$ with the multiplicity $l$. This shows that we can replace $\lambda_{\mu}^{(l)}$ by $\lambda_{\mu^{\prime}}^{(l)}$, the other root of $P_{l}(\lambda, \eta)=0$.

In (3.3) for a fixed $\mu$ we get $\gamma_{l}$ linearly independent eigenvectors for $\lambda_{\mu}^{(l)}$ and for a fixed $\nu: 1 \leqq \nu \leqq \gamma_{l}$ we get $m_{l}$ orthogonal eigenvectors of each $\lambda_{\mu}^{(l)}\left(1 \leqq \mu \leqq m_{l}\right)$ of $A(\eta)$.

Lemma 3.3 (Orthogonalization). There exist $T_{l}^{(\nu)}\left(\lambda_{\mu}^{(l)}, \eta\right)$ in (3.3) for $\nu=$ $1,2, \cdots, \gamma_{l}$ as orthogonal eigenvectors for $\lambda_{\mu}^{(l)}$ with components homogeneous polynomials in $\lambda_{\mu}^{(l)}$ of degree at most $\gamma_{l}\left(m-\gamma_{l}\right)$.

Proof. We set $a_{\nu}=T_{l}^{(\nu)}\left(\lambda_{\mu}^{(l)}, \eta\right)$ and

$$
\begin{aligned}
& \tilde{a}_{1}=a_{1}, \\
& \tilde{a}_{2}=\left(\tilde{a}_{1}, \tilde{a}_{1}\right) a_{2}-\left(a_{2}, \tilde{a}_{1}\right) \tilde{a}_{1},
\end{aligned}
$$

where $(a, b)=\sum_{i=1}^{m} a_{i} \bar{b}_{i}$, and for $1 \leqq \kappa \leqq l$

$$
\tilde{a}_{\kappa}=\prod_{r=1}^{\kappa-1}\left(\tilde{a}_{r}, \tilde{a}_{r}\right) a_{\kappa}-\left[\sum_{s=1}^{\kappa-1}\left\{\prod_{\substack{r \neq z \\ 1 \leqq r \leqq \kappa-1}}\left(\tilde{a}_{r}, \tilde{a}_{r}\right)\right\}\left(a_{\kappa}, \tilde{a}_{s}\right) \tilde{a}_{s}\right] .
$$

Then for any $p, 1 \leqq p<\kappa$,

$$
\begin{aligned}
\left(\tilde{a}_{\kappa}, \tilde{a}_{p}\right) & =\left\{\prod_{r=1}^{\kappa-1}\left(\tilde{a}_{r}, \tilde{a}_{r}\right)\right\}\left(a_{\kappa}, \tilde{a}_{p}\right)-\left[\sum_{s=1}^{\kappa-1}\left\{\prod_{\substack{r \neq s \\
1 \leqq r \leqq \kappa-1}}\left(\tilde{a}_{r}, \tilde{a}_{r}\right)\right\}\left(a_{\kappa}, \tilde{a}_{s}\right)\left(\tilde{a}_{s}, \tilde{a}_{p}\right)\right] \\
& =\left\{\prod_{r=1}^{\kappa-1}\left(\tilde{a}_{r}, \tilde{a}_{r}\right)\right\}\left(a_{\kappa}, \tilde{a}_{p}\right)-\left\{\prod_{\substack{r \neq p \\
1 \leqq r \leqq \kappa-1}}\left(\tilde{a}_{r}, \tilde{a}_{r}\right)\right\}\left(a_{\kappa}, \tilde{a}_{p}\right)\left(\tilde{a}_{p}, \tilde{a}_{p}\right)=0
\end{aligned}
$$

holds and $a_{\kappa}$ also is an eigenvector for $\lambda_{\mu}^{(l)}$, of degree $\kappa\left(m-\gamma_{l}\right)$.

For every $l=1,2, \cdots, k$ we can repeat the same process to construct the eigenvectors $T_{l}^{(\nu)}\left(\lambda_{\mu}^{(l)}, \eta\right)$. Thus we get

Theorem 3.1. There exists a matrix $T$ of order $m$ whose column vectors are orthogonal to each other such that

$$
\begin{aligned}
A(\eta) T= & T \operatorname{diag}\left(\lambda_{1}^{(1)}, \cdots, \lambda_{m_{1}}^{(1)} \cdots\right. \\
& \left.\cdot \lambda_{1}^{(l)}, \cdots, \lambda_{m l}^{(l)} \cdots \lambda_{1}^{(l)}, \cdots, \lambda_{m_{l}}^{(l)}, \cdots, \lambda_{1}^{(k)}, \cdots, \lambda_{m_{k}}^{(k)}\right)=D
\end{aligned}
$$

holds. Here the sequence $\lambda_{1}^{(l)}, \cdots, \lambda_{m_{l}}^{(l)}, l=1,2, \cdots, k$, occurs $\gamma_{l}$ times, and the blocks of the column vectors $T_{l}, l=1,2, \cdots, k$, are

$$
\begin{aligned}
T_{l}= & \left(T_{l}^{(1)}\left(\lambda_{1}^{(l)}, \eta\right) \cdots T_{l}^{(1)}\left(\lambda_{m l}^{(l)}, \eta\right),\right. \\
& \left.T_{l}^{(2)}\left(\lambda_{1}^{(l)}, \eta\right), \cdots, T_{l}^{(2)}\left(\lambda_{m_{l}}^{(l)}, \eta\right), \cdots, T_{l}^{\left(\gamma_{l}\right)}\left(\lambda_{1}^{(l)}, \eta\right), \cdots, T_{l}^{\left(\gamma_{l}\right)}\left(\lambda_{m_{p}}^{(l)}, \eta\right)\right), \\
l= & 1,2, \cdots, k,
\end{aligned}
$$

that is $T=\left(T_{1}, T_{2}, \cdots, T_{k}\right)$. 
4. Existence of partial lacunas. We transform the equation (2.1) by the $N$-dimensional Fourier transform

$$
\tilde{u}=\tilde{u}(t, \eta)=\frac{1}{(2 \pi)^{N / 2}} \int_{E_{N}} \exp (i x \cdot \eta) u(t, x) d x
$$

to

$$
\frac{\partial \tilde{u}}{\partial t}=-i A(\eta) \tilde{u}
$$

Let $v$ be an $m$-vector function $v(t, \eta)=\left(v_{i}(t, \eta) \cdot i \downarrow 1,2, \cdots, m\right)$. Divide its components into the groups just corresponding to the column-indices of $T$ in (3.5); that is

$$
\begin{aligned}
v_{l}^{(\nu)} & =\left(v_{l \mu}^{(\nu)}(t, \eta) \cdot \mu \downarrow 1,2, \cdots, m_{l}\right), \\
v_{l} & =\left(v_{l}^{(\nu)} \cdot \nu \downarrow 1, \cdots, \gamma_{l}\right), \\
v & =\left(v_{l} \cdot l \downarrow 1,2, \cdots, k\right) .
\end{aligned}
$$

Let $\left|T_{l}^{(\nu)}\left(\lambda_{\mu}^{(l)}, \eta\right)\right|=s_{l, \mu}^{(\nu)}$ be the Euclidean length of the vector $T_{l}^{(\nu)}\left(\lambda_{\mu}^{(l)}, \eta\right)$ and denote the m-vector function whose components are $v_{l \mu}^{(\nu)} / s_{l, \mu}^{(\nu) 2}$ by $v / s^{2}$. We next define a new variable $v$ by setting

$$
\tilde{u}=T \cdot \frac{v}{s^{2}} ; \quad v=T^{*} \cdot \tilde{u} .
$$

Note that $T$ is not normalized, hence $T^{*} T=\operatorname{diag}\left(s_{l \mu}^{(\nu) 2}\right)$, where $T^{*}={ }^{t} \bar{T}$.

In component form, these read

$$
\begin{aligned}
\tilde{u}_{i} & =\sum_{l} \sum_{\nu} \sum_{\mu} T_{l, i}^{(\nu)}\left(\lambda_{\mu}^{(l)}, \eta\right) \frac{v_{l \mu}^{(\nu)}}{s_{l \mu}^{(\nu) 2}} ; \\
v_{l \mu}^{(\nu)} & =\sum_{i=1}^{m} T_{l, i}^{(\nu)}\left(\lambda_{\mu}^{(l)}, \eta\right) \tilde{u}_{i} .
\end{aligned}
$$

The transformed differential equation of (4.2) by (4.3) is

$$
\frac{\partial v}{\partial t}=-i D v \text {. }
$$

$D$ is defined by the right hand side of (3.4), where the $l$-th block of (4.5) is the system

$$
\frac{\partial v_{l \mu}^{(\nu)}}{\partial t}=-i \lambda_{\mu}^{(l)} v_{l \mu}^{(\nu)}, \quad \mu \downarrow 1,2, \cdots, m_{l}, \quad \nu \downarrow 1,2, \cdots, \gamma_{l} .
$$

Next we shall reduce algebraically the components $T_{l i}^{(\nu)}(\lambda, \eta)$ modulo the polynomial $P_{l}(\lambda, \eta)$ in $\lambda$. Thus there are obtained $m \times \gamma_{l}$ expressions, which we denote by $P_{l i}^{(\nu)}(\lambda, \eta)$, that are polynomials in $\lambda$ of degree less than $m_{l}$, namely 


$$
\begin{aligned}
T_{l i}^{(\nu)}(\lambda, \eta)=P_{l i}^{(\nu)}(\lambda, \eta)=\sum_{\kappa=1}^{m l} p_{l i k}^{(\nu)}(\eta) \lambda^{\kappa-1}, & \\
& i \downarrow 1,2, \cdots, m, \nu \downarrow 1,2, \cdots, \gamma_{\iota} .
\end{aligned}
$$

The degree of $P_{l i k}^{(v)}(\eta)$ in $\eta$ is at most $\gamma_{l}\left(m-\gamma_{l}\right)-(\kappa-1)$. The matrix $T$ can then be expressed as

$$
T=P \cdot \Lambda
$$

where we set

$$
P=\left(p_{l i \kappa}^{(\nu)}(\eta):{ }_{\kappa}^{i} \underset{\downarrow}{\downarrow} 1,2, \cdots, \cdots, m_{l}, \nu \rightarrow 1,2, \cdots, \gamma_{l}, l \rightarrow 1,2, \cdots, k\right),
$$

and

$$
\Lambda=\operatorname{diag}(D_{1}, D_{2}, D_{2}, \cdots, \underbrace{D_{l}, D_{l}, \cdots, D_{l}}_{\gamma_{l}}, \cdots, D_{k})
$$

with

$$
D_{l}=\left(\begin{array}{cccc}
1 & 1 & \cdots & 1 \\
\lambda_{1}^{(l)} & \lambda_{2}^{(l)} & \cdots & \lambda_{m l}^{(l)} \\
\vdots & & & \\
\lambda_{1}^{(l) m l-1} & \lambda_{2}^{(l) m l-1} & \cdots & \lambda_{m l}^{(l) m l-1}
\end{array}\right) .
$$

Note that $\operatorname{det} \mathscr{D}_{l}$ is a Vandermonde determinant which does not vanish since $\lambda_{1}^{(l)}, \cdots, \lambda_{m l}^{(l)}$ are distinct.

Next we consider the equation

$$
P_{l}\left(\frac{\partial}{\partial t}, \frac{\partial}{\partial x}\right) w_{l, 1}^{(\nu)}=\left[\left(\frac{\partial}{\partial t}\right)^{m_{l}}+\sum_{s=0}^{m l} a_{l s}\left(\frac{\partial}{\partial x}\right)\left(\frac{\partial}{\partial t}\right)^{m l-s}\right] w_{l, 1}^{(\nu)}=0 .
$$

Here the $a_{l_{s}}(\partial / \partial x)$ are of course polynomials of degree $s$ in the variable $(\partial / \partial x)$. This is transformed by the Fourier transform into

$$
P_{l}\left(\frac{\partial}{\partial t},-i \eta\right) \tilde{w}_{l, 1}^{(\nu)}=0
$$

We shall introduce the new variables

$$
\frac{\partial \tilde{w}_{l, j}^{(p)}}{\partial t}=i \tilde{w}_{l, j+1}^{(p)}, \quad j \downarrow 1,2, \cdots, m_{l}-1,
$$

and thus (4.10) may be transformed into the system

$$
\frac{\partial}{\partial t}\left(\tilde{w}_{l, i}^{(\nu)} \cdot j \downarrow 1,2, \cdots, m_{l}\right)=-i \odot_{l} \cdot\left(\tilde{w}_{l, j}^{(\nu)} \cdot j \downarrow 1,2, \cdots, m_{l}\right),
$$


where

$$
P_{l}=\left(\begin{array}{cccccc}
0 & 1 & 0 & & \cdots & 0 \\
0 & 0 & 1 & 0 & \cdots & 0 \\
0 & & & \ddots & 0 \\
& & & & 1 \\
-a_{l, m l} & -a_{l, m_{l}-1} & \cdots & -a_{l, 1}
\end{array}\right) \text { and } a_{l, s}=a_{l, s}(-i \eta)
$$

Next we can show the system (4.12) is transformed into the system (4.6) by

$$
\tilde{w}_{l i}^{(\nu)}=\sum_{\mu=1}^{m_{l}} \lambda_{\mu}^{(l) i-1} v_{l \mu}^{(\nu)}
$$

or

$$
\left(\tilde{w}_{l i}^{(v)} \cdot i \downarrow 1,2, \cdots, m_{l}\right)=D_{l} \cdot\left(v_{i i}^{(v)} \cdot j \downarrow 1,2, \cdots, m_{l}\right)
$$

because

$$
\mathfrak{D}_{l}^{-1} \cdot \mathscr{P}_{l} \cdot \mathfrak{D}_{l}=\operatorname{diag}\left(\lambda_{1}^{(l)}, \lambda_{2}^{(l)}, \cdots, \lambda_{m l}^{(l)}\right) .
$$

But we can express $\tilde{w}_{l i}^{v}$ in terms of $\tilde{u}_{i}$ using (4.13), (4.4) and (4.7); that is

$$
\begin{aligned}
\tilde{w}_{l i}^{(\nu)} & =\sum_{\mu=1}^{m l} \lambda_{\mu}^{(l) i-1} v_{l \mu}^{(\nu)} \\
& =\sum_{\mu=1}^{m l} \lambda_{\mu}^{(\nu) i-1} \sum_{j=1}^{m} T_{l i}^{(\nu)}\left(\lambda_{\mu}^{(l)}, \eta\right) \tilde{u}_{i} \\
& =\sum_{\mu=1}^{m l} \sum_{j=1}^{m} \sum_{k=1}^{m_{l}} \lambda_{j}^{(l) i+k-2} p_{l j k}^{(\nu)}(\eta) \tilde{u}_{i} \\
& =\sum_{j=1}^{m} q_{l, i, j}^{(\nu)}(\eta) \tilde{u}_{j}
\end{aligned}
$$

where

$$
q_{l i j}^{(\nu)}(\eta)=\sum_{\mu, \kappa=1}^{l} \lambda_{\mu}^{(l) i+\kappa-2} p_{l j \kappa}^{(\nu)}(\eta)
$$

This polynomial is symmetric in the roots $\lambda_{j}^{(l)}, j=1, \cdots, m_{l}$, and because the leading coefficient of $P_{l}(\lambda, \eta)$ is one, is therefore a rational homogeneous polynomial function of the $\eta$. Since $Q=\Lambda \cdot T^{*}$ we can also see that the matrix

$$
Q=\left(q_{l i j}^{(\nu)}(\eta): \begin{array}{l}
i \downarrow 1,2, \cdots, m_{l} \\
j \rightarrow 1,2, \cdots, m
\end{array}, \nu \downarrow 1,2, \cdots, \gamma_{l}, l \downarrow 1,2, \cdots, k\right)
$$

is non-singular except on the algebraic set $A=\{\eta:|\mathfrak{A}|=0\}$ defined in Lemma 3.1 .

Thus we can express the solution $w_{l, i}^{(\nu)}$ of $(4.10)$ in the form (4.14). 
As we have

$P_{l}\left(\frac{\partial}{\partial t},-i \eta\right) \tilde{w}_{l, \mu}^{(\nu)}=P_{l}\left(\frac{\partial}{\partial t},-i \eta\right)\left(i \frac{d}{d t}\right)^{\mu-1} \tilde{w}_{l, 1}^{(\nu)}=\left(i \frac{d}{d t}\right)^{\mu-1} P_{l}\left(\frac{\partial}{\partial t},-i \eta\right) \tilde{w}_{l, 1}^{(\nu)}=0$,

it follows that $\tilde{w}_{l, \mu}^{(\nu)}\left(\mu=2, \cdots, m_{l}\right)$ are also solutions.

Remark. If $\tilde{w}_{l, i}^{(\nu)}=e_{l}^{(\nu)}(\eta) \widetilde{w}_{l, i}^{(\nu)}$, where $e_{l}^{(\nu)}(\eta)$ is a polynomial such that $e_{l}^{(\nu)}(\eta) \neq 0$ for nonzero real vectors $\eta, \widetilde{\widetilde{w}}_{l, i}^{(\nu)}$ also satisfies the above equation.

Taking the inverse Fourier transform of (4.14) we obtain

$$
w_{l, k}^{(\nu)}(x, t)=(i)^{c} \sum_{i=1}^{m} q_{l k j}^{(\nu)}\left(\frac{\partial}{\partial x}\right) u_{i}, \quad k=1, \cdots, m_{l},
$$

which satisfy (4.9), and then

$$
P_{l}\left(\frac{\partial}{\partial t}, \frac{\partial}{\partial x}\right) w_{l, k}^{(\nu)}(x, t)=0 .
$$

The expressions $w_{l, \mu}^{(v)}(x, t)$ are the differential lacunary components that we seek.

Theorem 4.1. To every irreducible factor $P_{l}(\lambda, \eta)$ of degree $m_{l}$ and of multiplicity $l$ in (2.5) there correspond $l$ groups of $m_{l}$ independent linear combinations of the d.l.c. $\left\{w_{l, \mu}^{(\nu)}(x, t) \cdot \mu \downarrow 1, \cdots, m_{l}\right\}, \nu=1, \cdots, \gamma_{l}$, of the derivatives of the $u_{i}$ of order at most $\gamma_{l}\left(m-\gamma_{l}\right)+m_{l}-1$ which satisfy the hyperbolic factor equation (4.18), and whose domain of dependence is therefore that of (4.18).

We note from the almost non-singular nature of the polynomial matrix $Q$ that if all $m$ variables $w_{l, \mu}^{(\nu)}$ corresponding to all irreducible factors $P_{l}(\lambda, \eta)$, are zero, then the solution $u_{i}$ is also zero. The system (4.17) is, by the same token, an almost elliptic system in the space variables, for the $u_{i}$.

Definition 4.1. A region is called a lacuna of the system (2.1) if it is one of the regions which is formed by the wave surface $W(t, x)$, and the value of the elementary solution for the Cauchy problem vanishes identically in that region.

Corollary 4.1. A lacuna for a factor $P_{l}(\lambda, \eta)$ is a lacuna for the linear combination $w_{l, \mu}^{(\nu)}(t, x)$ of Theorem 4.1 .

Thus we can show the existence of a partial lacuna of the system (2.1), in the following sense (see also [6], p. 48). While the values of the solution components $u_{i}(t, x)$ may still depend on the date in the lacuna region, the values of the differential lacunary components $w_{l, \mu}^{(\nu)}(t, x)$ are independent of the data in that region. Equivalently, the corresponding differential expression of components of the matrix elementary solution of (2.1) vanishes in the partial lacuna, although the elementary solution itself need not vanish there. We can also say that the d.l.c. $w_{l, \mu}^{(\nu)}(t, x)$ propagate only with the speed associated with the hyperbolic factor $P_{l}(\lambda, \mu)$ (see [22]). 
5. Cancellation operators. We next show that the set of differential lacunary components constructed in the preceding section are, in a certain sense to be defined, formed in the simplest possible way from the solution components. For this purpose we introduce the idea of a "cancellation operator".

Definition 5.1. An $m \times m$ matrix operator

$$
R\left(\frac{\partial}{\partial x}\right)=\left(R_{\imath}^{(\nu)}\left(\frac{\partial}{\partial x}\right): \nu \downarrow 1,2, \cdots, \gamma_{\imath}, l \rightarrow 1,2, \cdots, k\right),
$$

where

$$
R_{l}^{(\nu)}\left(\frac{\partial}{\partial x}\right)=\left(R_{l i j}^{(\nu)}\left(\frac{\partial}{\partial x}\right): \begin{array}{l}
i \downarrow 1,2, \cdots, m_{l} \\
j \rightarrow 1,2, \cdots, m
\end{array}\right)
$$

and all elements $R_{l i j}^{(\nu)}(\partial / \partial x)$ are partial differential operators of $x$ variables with constant coefficients, will be called a cancellation operator for the supp $u$, if each component $Z_{l \mu}^{(\nu)}$ of the block $Z_{l}^{(\nu)}=R_{l}^{(\nu)}(\partial / \partial x) u$ of the vector $Z=R(\partial / \partial x) u$ satisfies the equation $P_{l}(\partial / \partial t, \partial / \partial x) Z_{l \mu}^{(\nu)}=0, \mu=1,2, \cdots, m_{l}$.

Consequently the domain of dependence of $Z_{l \mu}^{(\nu)}$ is contained in that of $P_{l}(\lambda, \mu)$. The matrix operator $Q$ in (4.16) is a cancellation operator.

Theorem 5.1 (Minimal property of $Q)$. Let $R(\partial / \partial x)$ be a cancellation operator for the supp $u$. Then there exist a scalar polynomial $\pi(-i \eta)$ of $\eta$ and an $m \times m$ polynomial matrix $S_{1}(-i \eta)$ such that

$$
\pi\left(\frac{\partial}{\partial x}\right) R\left(\frac{\partial}{\partial x}\right)=S_{1}\left(\frac{\partial}{\partial x}\right) Q\left(\frac{\partial}{\partial x}\right)
$$

Proof. First we shall show

$$
\tilde{w}_{l}^{(\nu)}=\widetilde{q_{l}^{(\nu)}\left(\frac{\partial}{\partial x}\right)} u=q_{l}^{(\nu)}(-i \eta) \tilde{u}
$$

forms a system of fundamental solutions of the ordinary differential equation with the parameter $\eta$

$$
P_{l}\left(\frac{d}{d t}-i \eta\right) \tilde{w}=0
$$

By (4.13) $\tilde{w}_{l}^{(\nu)}=\mathfrak{D}_{l} v$, where $v=\left(v_{i} \cdot j \downarrow 1,2, \cdots, m_{l}\right)$ satisfy $(d / d t) v_{i}(t, \eta)=$ $-i \lambda_{j} v_{j}(t, \eta)$. The Wronskian of $\tilde{w}^{(\nu)}$ is

$$
\begin{aligned}
\bar{W}\left[\tilde{w}_{l}^{(\nu)}\right] & =\left|\begin{array}{cccc}
w_{l_{1}}^{(\nu)} & \tilde{w}_{l_{1}}^{(\nu)^{\prime}} & \cdots & \tilde{w}_{l_{1}}^{(\nu)\left(m_{l}-1\right)} \\
\vdots & \vdots & & \vdots \\
\tilde{w}_{l m l}^{(\nu)} & \tilde{w}_{l m_{l}}^{(\nu)} & \cdots & \tilde{w}_{l m_{l}}^{(\nu)\left(m_{l}-1\right)}
\end{array}\right|=\left|D_{l}\right|\left|\begin{array}{cccc}
v_{1} & v_{1}^{\prime} & \cdots & v_{1}^{\left(m_{l}-1\right)} \\
\vdots & \vdots & & \vdots \\
v_{m_{l}} & v_{m_{l}}^{\prime} & \cdots & v_{m_{l}}^{\left(m_{l}-1\right)}
\end{array}\right| \\
& =\left|D_{l}\right| \bar{W}[v]=\left|D_{l}\right|^{2}(-i)^{(1 / 2) m_{l}\left(m_{l}-1\right)} v_{1} v_{2} \cdots v_{m_{l}} .
\end{aligned}
$$


On the other hand, as $v_{j}(t, \eta)=C_{i}(\eta)^{-i \lambda_{j} t}$, we have

$$
\bar{W}\left[w_{l}^{(\nu)}\right]=\left|D_{l}\right|^{2}(-i)^{(1 / 2) m_{l}\left(m_{l}-1\right)} C_{1}(\eta) \cdots C_{m_{l}}(\eta) e^{-i\left(\lambda_{1}+\cdots+\lambda_{m}\right) t}
$$

which is not zero because we can choose $C_{j}(\eta) \neq 0$. And $P_{l}(d / d t,-i \eta)$ is an ordinary differential operator of normal form. Therefore, by the Cauchy uniqueness theorem $\tilde{Z}_{l_{\mu}}^{(\nu)}(t, \eta)$ are represented in linear combinations of $\tilde{w}_{l j}^{(\nu)}$,

$$
\tilde{Z}_{l \mu}^{(\nu)}(t, \eta)=\sum_{i=1}^{m l} s_{l \mu j}(-i \eta) \tilde{w}_{l j}^{(\nu)}(t, \eta), \quad \mu=1,2, \cdots, m,
$$

in the matrix form

$$
\widetilde{Z}_{l}=S_{l}^{(\nu)}(-i \eta) w_{l}^{(\nu)}=S_{l}^{(\nu)}(-i \eta) q_{l}^{(\nu)}(-i \eta) \tilde{u}(t, \eta)
$$

where

$$
S_{l}^{(\nu)}(-i \eta)=\left(s_{l \mu j}(-i \eta): \begin{array}{l}
\mu \downarrow 1,2, \cdots, m_{l} \\
j \rightarrow 1,2, \ldots, m_{l}
\end{array}\right) .
$$

Now we form an $m \times m$ matrix $S(-i \eta)$ as the following;

$$
S(-i \eta)=\operatorname{diag}\left\{S_{l}^{(\nu)}(-i \eta) \cdot \nu=1,2, \cdots, \gamma_{l}, l=1,2, \cdots, k\right\} .
$$

After we try this process for $\nu=1,2, \cdots, \gamma_{l}, l=1,2, \cdots, k$, we can set

$$
R(-i \eta)=S(-i \eta) Q(-i \eta) \text {. }
$$

By the non-singularity of $Q(-i \eta)$ except on the algebraic set $A$ we can see that the elements of $S(-i \eta)$ are rational functions of $\eta$. Therefore, we can choose a suitable polynomial $\pi(-i \eta)$ of $\eta$ such that the elements of the matrix

$$
S_{1}(-i \eta)=\pi(-i \eta) S(-i \eta)
$$

are polynomials in $\eta$. This shows

$$
\pi(-i \eta) R(-i \eta)=S_{1}(-i \eta) Q(-i \eta)
$$

which proves Theorem 5.1.

6. Supports and singular supports. In this section we shall study the relation between the supports and singular supports of the solution components and those of the d.l.c. The results will be stated in Theorem 6.2, for which purpose we shall extend the notions of $P$-convexity and strong P-convexity of [14] (also we may make reference to [21]) for a matrix operator. As for the method we reduce a system of equations to a single equation by multiplying by a cofactor matrix also used in [1] and [9] for other problems.

Lemma 6.1. Let $A, B$ be non-void subsets of $R^{N}$. Then if c.h. denotes convex hull we have

$$
\text { c.h. }(A \cup B)=\cup\{[x: y]: x \varepsilon \text { c.h. }(A), y \varepsilon \text { c.h. }(B)\}
$$


where

$$
[x: y]=\{z: z=a x+(1-a) y, 0 \leqq a \leqq 1\} .
$$

In particular we have

$$
\text { c. h. }(A \cup B)=\text { c. h. (c. h. }(A) \cup \text { c. h. }(B)) \text {. }
$$

Proof. We set $C=$ c. h. $(A \cup B)$. To show $\subseteq$, we observe that any $z \varepsilon C$ can be written $z=a x+(1-a) y$ for $x, y \varepsilon A \cup B, 0 \leqq a \leqq 1$, where cases occur as follows: (a) $x \varepsilon A, y \varepsilon A$, (b) $x \varepsilon A, y \varepsilon B$ or $x \varepsilon B, y \varepsilon A$, (c) $x \varepsilon B, y \varepsilon B$. In the case (a), $z \varepsilon$ c.h. (A); this is the case for $a=1$, and the case (c) corresponds to $a=0$ in the right-hand side. The case (b) is obvious. To show $\supseteq$, we have $x \varepsilon$ c.h. $(A) \subseteq$ c.h. $(A \cup B)$ and $y$ \& c.h. $(B) \subseteq$ c.h. $(A \cup B)$. Therefore, $z=a x+(1-a) y, 0 \leqq a \leqq 1, \varepsilon$ c.h. $(A \cup B)$. The second part follows from

$$
\text { c.h. (c.h. }(A) \cup \text { c.h. }(B))=\bigcup\{[x: y]: x \varepsilon \text { c.h. }(A), y \varepsilon \text { c.h. }(B)\} \text {. }
$$

Now we can state

\section{Theorem 6.1. Let}

$$
P\left(\frac{\partial}{\partial x}\right)=\left(P_{i j} \frac{\partial}{((\partial x))}: \begin{array}{l}
i \downarrow 1,2, \cdots, m \\
j \rightarrow 1,2, \cdots, m
\end{array}\right)
$$

be an $m \times m$ matrix of partial differential operators with constant coefficients and $\mu=\left(\mu_{i} \cdot j \downarrow 1,2, \cdots, m\right) \varepsilon\left(\mathcal{E}^{\prime}\left(R^{N}\right)\right)^{m}$. Set $f=\left(f_{i} \cdot j \downarrow 1,2, \cdots, m\right)=P \mu$. Then we have

$$
\text { c.h. } \bigcup_{i=1}^{m} \operatorname{supp} \mu_{i}=\text { c.h. } \bigcup_{i=1}^{m} \operatorname{supp} f_{i}
$$

$$
\text { c.h. } \bigcup_{i=1}^{m} \operatorname{sing} \operatorname{supp} \mu_{i}=\text { c.h. } \bigcup_{i=1}^{m} \operatorname{sing} \operatorname{supp} f_{i} \text {. }
$$

Proof. (a) Let $P(-i \eta)$ be the $m \times m$ matrix whose elements are the cofactors of the corresponding entries in the transpose of $P(-i \eta)$, denoted by

$$
\check{P}=\left(P^{k l}: \begin{array}{l}
k \downarrow 1,2, \cdots, m \\
l \rightarrow 1,2, \cdots, m
\end{array}\right) .
$$

Multiplying $\check{P}$ on $P_{\mu}=f$ we have

$$
\left|P\left(\frac{\partial}{\partial x}\right)\right| \mu_{k}=\sum_{l=1}^{m} P^{k l} f_{l}, \quad k \downarrow 1,2, \cdots, m .
$$

By the $|P|$ - and $P^{k l}$-convexity of $R_{0}^{N}$ and Lemma 6.1 we have

$$
\text { c.h. } \begin{aligned}
\left(\operatorname{supp} \mu_{k}\right) & =\text { c.h. }\left(\operatorname{supp} \sum_{l=1}^{m} P^{k l} f_{l}\right) \subseteq \text { c.h. } \bigcup_{l=1}^{m}\left(\operatorname{supp} P^{k l} f_{l}\right) \\
& =\text { c.h. } \bigcup_{l=1}^{m} \text { c.h. }\left(\operatorname{supp} P^{k l} f_{l}\right)=\text { c.h. } \bigcup_{l=1}^{m} \text { c.h. }\left(\operatorname{supp} f_{l}\right) \\
& =\text { c.h. } \bigcup_{l=1}^{m} \operatorname{supp} f_{l} .
\end{aligned}
$$


Therefore we have

$$
\bigcup_{i=1}^{m} \text { c.h. }\left(\operatorname{supp} \mu_{i}\right) \subseteq \text { c.h. } \bigcup_{i=1}^{m} \operatorname{supp} f_{i} .
$$

Hence we have

$$
\text { c.h. } \bigcup_{i=1}^{m} \text { c.h. }\left(\operatorname{supp} \mu_{i}\right) \subseteq \text { c.h. } \bigcup_{i=1}^{m} \operatorname{supp} f_{i} \text {. }
$$

Using again Lemma 6.1 we have

$$
\text { c.h. } \bigcup_{i=1}^{m} \operatorname{supp} \mu_{i} \subseteq \text { c.h. } \bigcup_{i=1}^{m} \operatorname{supp} f_{i} \text {. }
$$

On the other hand, as $f_{i}=\sum_{j=1}^{m} P_{i j}(\partial / \partial x) \mu_{i}$, we have

$$
\operatorname{supp} f_{i}=\operatorname{supp}\left(\sum_{i=1}^{m} P_{i i}\left(\frac{\partial}{\partial x}\right) \mu_{i}\right) \subseteq \bigcup_{i=1}^{m} \operatorname{supp} \mu_{i} ;
$$

that is

$$
\text { c.h. } \bigcup_{i=1}^{m} \operatorname{supp} f_{i} \subseteq \text { c.h. } \bigcup_{j=1}^{m} \operatorname{supp} \mu_{i} \text {. }
$$

(6.1) and (6.2) prove (a). The proof of (b) can be obtained by replacing supp for sing supp in that of (a) because $R^{N}$ is also strongly $|P|$ - and $P^{k l}$-convex.

Next we shall note in some cases other stronger relations between the solution components and the d.l.c. can be found which lead to a stronger result on their singular supports. To state this we use (see [5], p. 505, [13], p. 528).

Definition 6.1. An $m^{\prime} \times m^{\prime}$ matrix $P^{\prime}(\partial / \partial x)$ of partial differential operators with constant coefficients is called an elliptic system if there exist pairs of integers $\left(t_{k}, s_{j}\right), j, k=1,2, \cdots, m^{\prime}$, such that

(a) order of $P_{j k}(\partial / \partial x) \leqq t_{k}-s_{i}$, and

(b) $\operatorname{det}\left(P_{j k}^{0}(-i \eta)\right) \neq 0$ for any real vector $\eta \neq 0$, where $P_{j k}^{0}(-i \eta)$ is the part of $P_{j k}(-i \eta)$ with order exactly $t_{k}-s_{j}$.

Definition 6.2. An $m^{\prime} \times m^{\prime \prime}\left(m^{\prime}<m^{\prime \prime}\right)$ matrix $Q^{\prime}(\partial / \partial x)$ of partial differential operators with constant coefficients is called a hypoelliptic system if every distribution solution $\mu=\left(\mu_{i} \cdot i \downarrow 1, \cdots, m^{\prime}\right)$ of $Q^{\prime}(\partial / \partial x) \mu=f$ is in $C^{\infty}$ in every open set where $f=\left(f_{i} \cdot j \downarrow 1, \cdots, m^{\prime \prime}\right)$ is in $C^{\infty}$.

This property was characterized in [13; p. 530, Corollary], as the following: The distance from the real point $\eta$ to the set of the complex $N$-plane where the rank of $Q^{\prime}(\zeta)<m^{\prime}$ tends to infinity with $\eta$.

Theorem 6.2. Let $P^{\prime}(\partial / \partial x)$ be an $m^{\prime} \times m^{\prime}$ elliptic system and $Q^{\prime}(\partial / \partial x)$ be an $m^{\prime} \times m^{\prime \prime}\left(m^{\prime}<m^{\prime \prime}\right)$ hypoelliptic system and let $\mu \varepsilon\left[\mathcal{E}^{\prime}\left(R^{N}\right)\right]^{m^{\prime}}$ and $f \varepsilon\left[\mathcal{E}^{\prime}\left(R^{N}\right)\right]^{m^{\prime \prime}}$ 
satisfy $P^{\prime} \mu=Q^{\prime} f$. Then we have

$$
\bigcup_{i=1}^{m^{\prime}} \operatorname{sing} \operatorname{supp} \mu_{i}=\bigcup_{i=1}^{m^{\prime \prime}} \operatorname{sing} \operatorname{supp} f_{i} \text {. }
$$

Proof. Choose an open set $\Omega$ containing all supports of $\mu_{i}$ and $f_{i}$. Let $\Omega_{i}$ and $\omega_{i}$ be the unions of open subsets of $\Omega$ where $\mu_{i}$ and $f_{i}$ are $C^{\infty}$ respectively. By "the theorem of interior regularity of solutions of elliptic system" (see [5], Theorem 4, p. 529), we have

$$
\bigcap_{i=1}^{m^{\prime}} \omega_{i} \subseteq \bigcap_{i=1}^{m} \Omega_{i}
$$

On the other hand by the hypoellipticity of $Q^{\prime}$ the inverse inclusion is valid. Hence we have

$$
\bigcap_{j=1}^{m^{\prime}} \omega_{i}=\bigcap_{i=1}^{m} \Omega_{i}
$$

Taking the complements of both sides we find the conclusion.

It follows that in Theorem 6.1 we may take $P$ as the cancellation operator, $\mu$ as the solution vector $u$ and $f$ as the differential lacunary vector $w$ for any solution of the hyperbolic system (2.1). Observe that if the $w_{i}$ all vanish identically, then c.h. supp $w_{i}=\varnothing$, and the solution $u_{i}$ is also identically zero because we may assume supp $u_{i}$ is compact for every fixed $t$. That is, the d.l.c. determines the entire solution uniquely through the solution of the system (4.17).

7. Examples. (1) Maxwell's equations in vacuo (see [4], p. 176). For $E=$ $\left(u_{i} \cdot i \downarrow 1,2,3\right), H=\left(u_{i} \cdot i \downarrow 4,5,6\right)$ this equation is

$$
\begin{gathered}
\frac{\partial E}{\partial t}=\operatorname{curl} H, \\
\frac{\partial H}{\partial t}=-\operatorname{curl} E, \text { with } \\
A(\eta)=\left(\begin{array}{rrrrrr}
0 & 0 & 0 & 0 & -\eta_{3} & \eta_{2} \\
0 & 0 & 0 & \eta_{3} & 0 & -\eta_{1} \\
0 & 0 & 0 & -\eta_{2} & \eta_{1} & 0 \\
0 & \eta_{3} & -\eta_{2} & 0 & 0 & 0 \\
-\eta_{3} & 0 & \eta_{1} & 0 & 0 & 0 \\
\eta_{2} & -\eta_{1} & 0 & 0 & 0 & 0
\end{array}\right)
\end{gathered}
$$

and $\Delta(\lambda)^{2}=\lambda^{2}\left(\lambda^{2}-|\eta|^{2}\right)^{2}$ where $|\eta|^{2}=\eta_{1}^{2}+\eta_{2}^{2}+\eta_{3}^{2}$. The irreducible factors are $P_{1}(\lambda, \eta)=\lambda$ and $P_{2}(\lambda, \eta)=\lambda^{2}-|\eta|_{2}$. We have $\gamma_{1}=\gamma_{2}=2$ and $\lambda_{1}^{(1)}=0$, $\lambda_{1}^{(2)}=|\eta|$ and $\lambda_{2}^{(2)}=-|\eta|$, and we can choose any one of the three cyclically 
related eigenvectors,

$$
T_{1}^{(1)}(\lambda, \eta)=T_{2}^{(1)}(\lambda, \eta)=\left(\begin{array}{c}
-\lambda \eta_{2} \\
\lambda \eta_{1} \\
0 \\
\eta_{1} \eta_{3} \\
\eta_{2} \eta_{3} \\
\eta_{3}^{2}-\lambda^{2}
\end{array}\right],\left(\begin{array}{c}
0 \\
-\lambda \eta_{3} \\
\lambda \eta_{2} \\
\eta_{1}^{2}-\lambda^{2} \\
\eta_{2} \eta_{1} \\
\eta_{3} \eta_{1}
\end{array}\right] \text { or }\left[\begin{array}{c}
\lambda \eta_{3} \\
0 \\
-\lambda \eta_{1} \\
\eta_{1} \eta_{2} \\
\eta_{2}^{2}-\lambda^{2} \\
\eta_{3} \eta_{2}
\end{array}\right]
$$

By using the special form of $A(\eta)$, namely the third order right upper block is equal to the negative of the left lower block, we have

$$
T_{1}^{(2)}(\lambda, \eta)=T_{2}^{(2)}(\lambda, \eta)=\left(\begin{array}{c}
\eta_{1} \eta_{2} \\
\eta_{2} \eta_{3} \\
\eta_{3}^{2}-\lambda^{2} \\
-\lambda \eta_{2} \\
\lambda \eta_{1} \\
0
\end{array}\right],\left(\begin{array}{c}
\eta_{1}^{2}-\lambda^{2} \\
\eta_{2} \eta_{1} \\
\eta_{3} \eta_{1} \\
0 \\
-\lambda \eta_{3} \\
\lambda \eta_{2}
\end{array}\right) \quad \text { or }\left[\begin{array}{c}
\eta_{1} \eta_{2} \\
\eta_{2}^{2}-\lambda^{2} \\
\eta_{3} \eta_{2} \\
\lambda \eta_{3} \\
0 \\
-\lambda \eta_{1}
\end{array}\right] .
$$

Rewriting these vectors modulo $P_{1}(\lambda, \eta)$ and $P_{2}(\lambda, \eta)$ respectively and reducing the degrees of them if possible we have for the first vectors among the three in each of the two given sets

$$
T=\left[\begin{array}{cccccc}
0 & \eta_{1} & -|\eta| \eta_{2} & |\eta| \eta_{2} & \eta_{1} \eta_{3} & \eta_{1} \eta_{3} \\
0 & \eta_{2} & |\eta| \eta_{1} & -|\eta| \eta_{1} & \eta_{2} \eta_{3} & \eta_{2} \eta_{3} \\
0 & \eta_{3} & 0 & 0 & \eta_{3}^{2}-|\eta|^{2} & \eta_{3}^{2}-|\eta|^{2} \\
\eta_{1} & 0 & \eta_{1} \eta_{3} & \eta_{1} \eta_{3} & |\eta| \eta_{2} & -|\eta| \eta_{2} \\
\eta_{2} & 0 & \eta_{2} \eta_{3} & \eta_{2} \eta_{3} & -|\eta| \eta_{1} & |\eta| \eta_{1} \\
\eta_{3} & 0 & \eta_{3}^{2}-|\eta|^{2} & \eta_{3}^{2}-|\eta|^{2} & 0 & 0
\end{array}\right]
$$

and

$$
Q=\left[\begin{array}{cccccc}
0 & 0 & 0 & \eta_{1} & \eta_{2} & \eta_{3} \\
\eta_{1} & \eta_{2} & \eta_{3} & 0 & 0 & 0 \\
0 & 0 & 0 & 2 \eta_{1} \eta_{3} & 2 \eta_{2} \eta_{3} & 2\left(\eta_{3}^{2}-|\eta|^{2}\right) \\
-2 \eta_{2}|\eta|^{2} & 2 \eta_{1}|\eta|^{2} & 0 & 0 & 0 & 0 \\
2 \eta_{1} \eta_{3} & 2 \eta_{2} \eta_{3} & 2\left(\eta_{3}^{2}-|\eta|^{2}\right) & 0 & 0 & 0 \\
0 & 0 & 0 & 2 \eta_{2}|\eta|^{2} & -2 \eta_{1}|\eta|^{2} & 0
\end{array}\right]
$$


indicating similar but cyclically permuted matrix forms $T$ and $Q$ for the second and third vectors of the sets of cyclically related eigenvectors given above. This shows the first and the second rows of $Q(\partial / \partial x) u$ are $i \operatorname{div} H$ and $i \operatorname{div} E$ which are the d.l.c. for the factor $P_{1}(\lambda, \eta)=\lambda$. Therefore their domains of dependence are the origin only. The third and fourth are the third components of -2 curl curl $H$ and $-2 i \Delta \cdot \operatorname{curl} E$, and the fifth and sixth are components of -2 curl curl $E$ (or $H$ ) and $-2 i \Delta \cdot \operatorname{curl} E$ (or $H$ ), which are all the d.l.c. for the factor $P_{2}(\lambda, \eta)=\lambda^{2}-|\eta|^{2}$. Here

$$
\Delta=\frac{\partial^{2}}{\partial x_{1}^{2}}+\frac{\partial^{2}}{\partial x_{2}^{2}}+\frac{\partial^{2}}{\partial x_{3}^{2}}
$$

Hence their domains of dependence are the surface of the unit sphere. While the solution vector $u=u(t, x)$ has the surface and interior of the sphere of radius $t$ as its domain of dependence (see [6], p. 54), and by the remark preceding (4.17), curl $E$ (or $H$ ) also is the d.l.c. As Theorem 6.1 asserts, the convex hulls of the sums of the supports for a fixed $t$ are identical. The other components of these vectors may be obtained by taking other matrix forms $T$.

To see how to apply Theorem 6.2 we set, for example, $w_{0}=\operatorname{div} E, w_{i}=$ (curl $\operatorname{curl} E)_{i}=$ the $i$-th component of curl curl $E, w=\left(w_{i} \cdot i \downarrow 0,1,2,3\right)$,

$$
\begin{aligned}
& P^{\prime}\left(\frac{\partial}{\partial x}\right)=\left(\begin{array}{ccc}
\Delta & 0 & 0 \\
0 & \Delta & 0 \\
0 & 0 & \Delta
\end{array}\right) \text { : elliptic system, and } \\
& Q^{\prime}\left(\frac{\partial}{\partial x}\right)=\left(\begin{array}{rrrr}
\frac{\partial}{\partial x_{1}} & -1 & 0 & 0 \\
\frac{\partial}{\partial x_{2}} & 0 & -1 & 0 \\
\frac{\partial}{\partial x_{3}} & 0 & 0 & -1
\end{array}\right] \begin{array}{l}
\text { hypoelliptic system, } \\
\text { on account of rank } \\
Q^{\prime}(\zeta)=3 .
\end{array}
\end{aligned}
$$

Hence we have $P^{\prime}(\partial / \partial x) E=Q^{\prime}(\partial / \partial x) w$. It follows from Theorem 6.2 that $\bigcup_{i=1}^{3} \operatorname{sing} \operatorname{supp} u_{i}=\bigcup_{j=0}^{3} \operatorname{sing} \operatorname{supp} w_{i}$, while the differential lacunary property of $w$ shows that the singular set of the right hand side is the origin and the surface of the sphere of radius $t$.

(2) Acoustic equations. This is written for $v=\left(u_{i} \cdot i \downarrow 1,2,3\right), p=u_{4}$ and $u=\left(u_{i} \cdot i \downarrow 1,2,3,4\right)$,

$$
\begin{aligned}
& \frac{\partial}{\partial t} v=-\operatorname{grad} p \\
& \frac{\partial}{\partial t} p=-\operatorname{div} v
\end{aligned}
$$


Therefore we can take

$$
A(\eta)=\left(\begin{array}{rrrr}
0 & 0 & 0 & -\eta_{1} \\
0 & 0 & 0 & -\eta_{2} \\
0 & 0 & 0 & -\eta_{3} \\
-\eta_{1} & -\eta_{2} & -\eta_{3} & 0
\end{array}\right) \quad \text { where } \quad|\eta|^{2}=\eta_{1}^{2}+\eta_{2}^{2}+\eta_{3}^{2}
$$

We have $\lambda_{1}^{(1)}=0, \lambda_{2}^{(1)}=|\eta|, \lambda_{2}^{(2)}=-|\eta|, \gamma_{1}=2$ and $\gamma_{2}=1$. As a matrix $T$ we have

$$
\left(\begin{array}{cccc}
\eta_{2} & \eta_{1} \eta_{3} & -\eta_{1} & -\eta_{1} \\
-\eta_{1} & \eta_{2} \eta_{3} & -\eta_{2} & -\eta_{2} \\
0 & -\left(\eta_{1}^{2}+\eta_{2}^{2}\right) & -\eta_{3} & -\eta_{3} \\
0 & 0 & |\eta| & -|\eta|
\end{array}\right)
$$

then

$$
Q(\eta)=\Lambda \cdot T^{*}=\left(\begin{array}{cccc}
\eta_{2} & -\eta_{1} & 0 & 0 \\
\eta_{1} \eta_{3} & \eta_{2} \eta_{3} & -\left(\eta_{1}^{2}+\eta_{2}^{2}\right) & 0 \\
-2 \eta_{1} & -2 \eta_{2} & -2 \eta_{3} & 0 \\
0 & 0 & 0 & 2|\eta|^{2}
\end{array}\right)
$$

which shows that the third component of curl $v$ and curl curl $v$ are d.l.c. for $\lambda$. By taking other eigenvectors for $\lambda=0$, for example, some suitable permutation on entries of $T$, curl $v$ and curl curl $v$ are d.l.c. for $\lambda$. And div $v$ and $\Delta p$ are d.l.c. for $\lambda^{2}-|\eta|^{2}$. By the remark preceding (4.17), $p$ is also d.l.c. for $\lambda^{2}-|\eta|_{2}$. Hence similar consideration as (1) for domains of dependence of these d.l.c. and the solution components is possible. On their singular supports we set $w_{0}=\operatorname{grad}$ $v, w_{i}=(\operatorname{curl} \operatorname{curl} v)_{i}, i=1,2,3, w_{4}=\Delta p$ and $w=\left(w_{i} \cdot i \downarrow 1, \cdots, 4\right)$. We choose

$$
P^{\prime}\left(\frac{\partial}{\partial x}\right)=\left(\begin{array}{cccc}
\Delta & 0 & 0 & 0 \\
0 & \Delta & 0 & 0 \\
0 & 0 & \Delta & 0 \\
0 & 0 & 0 & \Delta
\end{array}\right) \text { and } Q^{\prime}\left(\frac{\partial}{\partial x}\right)=\left(\begin{array}{ccccc}
\frac{\partial}{\partial x_{1}} & -1 & 0 & 0 & 0 \\
\frac{\partial}{\partial x_{2}} & 0 & -1 & 0 & 0 \\
\frac{\partial}{\partial x_{3}} & 0 & 0 & -1 & 0 \\
0 & 0 & 0 & 0 & 1
\end{array}\right)
$$

which are an elliptic system and a hypoelliptic system respectively, and $P^{\prime} u=$ $Q^{\prime} w$ holds. This shows Theorem 6.2 is applicable to this case. 
(3) Dirac's equations with zero rest-mass (see [4], p. 179). This is written for $u=\left(u_{i} \cdot i \downarrow 1,2,3,4\right)$,

$$
\begin{aligned}
\alpha_{1}= & \left.\begin{array}{rrrr}
0 & 0 & 0 & 1 \\
0 & 0 & 1 & 0 \\
0 & 1 & 0 & 0 \\
1 & 0 & 0 & 0
\end{array}\right), & \alpha_{2}=\left[\begin{array}{rrrr}
0 & 0 & 0 & -i \\
0 & 0 & i & 0 \\
0 & -i & 0 & 0 \\
i & 0 & 0 & 0
\end{array}\right), \\
\alpha_{3}=\left(\begin{array}{rrrr}
0 & 0 & 1 & 0 \\
0 & 0 & 0 & -1 \\
1 & 0 & 0 & 0 \\
0 & -1 & 0 & 0
\end{array}\right] & \alpha_{4}= &
\end{aligned}
$$

and $a=\left(a_{i} \cdot i \downarrow 1,2,3,4\right)$,

$$
\sum_{k=1}^{4} \alpha_{k}\left(\frac{\partial}{\partial x_{k}}-a_{k}\right) u=0
$$

By setting $u=\exp \left(\sum_{k=1}^{4} a_{k} x_{k}\right) \cdot v$ we have from this equation

$$
\sum_{k=1}^{4} \alpha_{k} \frac{\partial}{\partial x_{k}} v=0
$$

Considering $x_{4}$ as the time variable we have

$$
A(\eta)=\left(\begin{array}{cccc}
0 & 0 & \eta_{3} & \eta_{1}-i \eta_{2} \\
0 & 0 & \eta_{1}+i \eta_{2} & -\eta_{3} \\
\eta_{3} & \eta_{1}-i \eta_{2} & 0 & 0 \\
\eta_{1}+i \eta_{2} & -\eta_{3} & 0 & 0
\end{array}\right)
$$

and $\Delta(\eta)=\left(\lambda^{2}-|\eta|^{2}\right)^{2}$, and

and

$$
T=\left[\begin{array}{cccc}
\eta_{3} & \eta_{3} & \eta_{1}-i \eta_{2} & \eta_{1}-i \eta_{2} \\
\eta_{1}+i \eta_{2} & \eta_{1}+i \eta_{2} & -\eta_{3} & -\eta_{3} \\
|\eta| & -|\eta| & 0 & 0 \\
0 & 0 & |\eta| & -|\eta|
\end{array}\right]
$$

These show that

$$
Q(\eta)=\left[\begin{array}{cccc}
2 \eta_{3} & 2\left(\eta_{1}-i \eta_{2}\right) & 0 & 0 \\
0 & 0 & 2|\eta|^{2} & 0 \\
2\left(\eta_{1}+i \eta_{2}\right) & -2 \eta_{3} & 0 & 0 \\
0 & 0 & 0 & 2|\eta|^{2}
\end{array}\right] .
$$

$$
\left[\frac{\partial}{\partial x_{3}} v_{1}+\left(\frac{\partial}{\partial x_{1}}-i \frac{\partial}{\partial x_{2}}\right) v_{2}\right], \Delta v_{3},\left[\left(\frac{\partial}{\partial x_{1}}+i \frac{\partial}{\partial x_{2}}\right) v_{1}-\frac{\partial}{\partial x_{3}} v_{2}\right]
$$


and $\Delta v_{4}$ are d.l.c. for $\lambda^{2}-|\eta|^{2}$. By the remark preceding (4.17), $v_{3}$ and $v_{4}$ are also the d.l.c. for $\lambda^{2}-|\eta|^{2}$. On their singular supports since $Q(\eta)$ is an elliptic system we may take $Q(\eta)=P^{\prime}(\eta)$ and $Q^{\prime}(\eta)=$ identity and have the result immediately that the singular support of the d.l.c. is the surface of the sphere of radius $t$.

\section{BIBLIOGRAPHY}

[1] S. Agmon, A. Douglis, L. Nirenberg, Estimates near the boundary for solutions of elliptic partial differential equations II, Comm. Pure Appl. Math., 17 (1964) 35-92.

[2] V. A. Bonovikov, Some sufficient conditions for lacunary lack of hyperbolic equations, Mat. Sb., 55 (1961) 237.

[3] V. A. Bonovikov, General formulae of Petrowski and Herglotz and diffusion of waves, Dokl. Akad. Nauk, 106 (1956) 587.

[4] R. Courant, D. Hilbert, Methods of Mathematical Physics II, Interscience, 1962.

[5] A. Douglis, L. Nirenberg, Interior estimates for elliptic systems, Comm. Pure Appl. Math., 8 (1955) 503-538.

[6] G. F. D. DuFf, On the Riemann Matrix of a Hyperbolic System, M. R. C. Report \#246, Madison, Wisconsin, 1961.

[7] G. F. D. Duff, The Cauchy problem for elastic waves in an antisotropic medium, Philos. Trans. Roy. Soc. London Ser. A., 252 (1960) 249-273.

[8] K. O. Friedrichs, Symmetric hyperbolic linear differential equations, Comm. Pure Appl. Math., 7 (1954) 345-392.

[9] B. FUGLEDE, A priori inequalities connected with systems of partial differential equations, Acta Math., 105 (1961) 177-195.

[10] S. A. Gal'pern, Lacunas of non-hyperbolic equations, Dokl. Akad. Nauk, 132 (1960), Soviet. Math. Dokl., 1 (1960) 680-683.

[11] P. GÜNтher, Einige Sätze über huygenssche Differentialgleichungen, Wiss. Z. KarlMarx-Univ. Leipzig, 14 (1965) 497-507.

[12] J. Hadamard, Lecture on Cauchy's Problem, Dover, 1952.

[13] L. HörmANDER, Differentiability properties of solutions of systems of differential equations, Ark. Mat., 3 (1958) 527-535.

[14] L. Hörmander, Linear Partial Differential Operators, Springer, 1963.

[15] F. John, Plane Waves and Spherical Means, Interscience, 1955.

[16] P. D. LAx, On Cauchy's problem for hyperbolic equations and differentiability of solutions of elliptic equations, Comm. Pure Appl. Math., 8 (1955) 615-633.

[17] J. Leray, Hyperbolic Differential Equations, Princeton, 1953.

[18] I. G. Perrowsky, On the diffusion of waves and the lacunas for hyperbolic equations, Math. Sb., 17 (1945) 289-370.

[19] I. G. Petrowsky, On the problem of Cauchy for a system of linear partial differential equations in the domain of non-analytic functions, Bull. Univ. d'Etat Moscow, 7 (1938) $1-74$.

[20] K. Steldmacher, Eine Klasse huygensscher Differentialgleichungen und ihre Integration, Math. Ann., 130 (1955) 219-233.

[21] F. Treves, Linear Partial Differential Equations with Constant Coefficients, Gordon and Breach, 1966.

[22] C. Truesdell, General and exact theory of waves in finite elastic strain, Arch. Rational Mech. Anal., 8 (1961), 263-296.

University of Toronto

Osaka University and University of Toronto

Date communicated: February 2, 1969 